Gastroenterologe 2016 $\cdot 11: 159$

DOI 10.1007/s11377-016-0070-z

Online publiziert: 19. April 2016

๑) Springer-Verlag Berlin Heidelberg 2016

CrossMark

Im Jahr 2013 erlagen etwa $25 \%$ der in Deutschland verstorbenen Patienten einem Krebsleiden. Damit bleibt Krebs mit 223.842 Sterbefällen nach den HerzKreislauf-Erkrankungen die zweithäufigste Todesursache. Das durchschnittliche Sterbealter der Krebserkrankten war mit 73,4 Jahren das höchste bislang gemessene. Der Anteil der mit Krebs verstorbenen Patienten, die 85 Jahre und älter waren, lag bei $17 \%$. Dies zeigt, dass Krebs zunehmend eine Erkrankung des fortgeschrittenen Alters ist. Des Weiteren leben Patienten heute deutlich länger mit der Diagnose Krebs und ein großer Teil der Patienten mit Krebserkrankungen kann heute geheilt werden.

Werden Krebserkrankungen im Frühstadium entdeckt, können sie meistens lokal behandelt werden, ohne dass dies $\mathrm{zu}$ langfristigen Einschränkungen führen wird. Im fortgeschrittenen Stadium sind häufig multimodale Therapiekonzepte erforderlich mit Chemotherapie und Bestrahlung sowie chirurgischen Eingriffen. Chemotherapieassoziierte Komplikationen treten häufig früh auf, sind vielseitig und zum Teil nicht reversibel. Frau Dr. Güthle und Herr Prof. Seufferlein fassen in ihrem Beitrag die therapiebedingten Spätfolgen und ihre Beeinflussung der Lebensqualität zusammen.

Bestrahlungsfolgen treten dagegen zum Teil akut und erst Jahre bzw. Jahrzehnte später auf und sind dann nicht einfach zu behandeln. Entstehen nach chirurgischer Therapie Defekte oder wird die Anatomie nach Resektion und Rekonstruktionen des Magen-Darm-Trakts deutlich verändert, kann dies langfristig

\author{
R. M. Schmid' • J. F. Riemann² \\ ${ }^{1}$ II. Medizinische Klinik und Poliklinik, München, Deutschland \\ ${ }^{2}$ Medizinische Klinik C, Klinikum Ludwigshafen, Stiftung LebensBlicke, Ludwigshafen, Deutschland
}

\title{
Leben mit und nach Krebs
}

bzw. dauerhaft zu Einschränkungen der Funktionen mit entsprechenden Nebenwirkungen und Einschränkungen der Lebensqualität führen. Herr Prof. Kreis und Herr PD Dr. Gröne geben einen umfassenden Überblick über die Folgezustände nach operativen Eingriffen bei gastrointestinalen Tumoren.

Alle Krebserkrankungen sind mit psychosozialen Belastungen verbunden. Das beginnt mit der Mitteilung der Diagnose, dem Eintreten von Nebenwirkungen der Therapie und der Feststellung eines Rezidivs. Herr Dr. Giesler und Herr Prof. Weis geben in ihrem Beitrag einen Überblick über die komplexe Bestimmung des psychoonkologischen Unterstützungsbedarfs.

Eine strukturierte Nachsorge ist bislang nur für wenige Entitäten in S3-Leitlinien integriert, wie Prof. Plentz und Prof. Malek in ihrem Beitrag ausführen. Wichtig in diesem Zusammenhang ist neben der Primär- und Sekundärprävention auch die Tertiärprävention v. a. für das Kolonkarzinom. Die Stiftung LebensBlicke leistet dazu einen ganz wichtigen Beitrag, wie von Prof. Ebert und Prof. Riemann ausgeführt. Sie hat sich zum Ziel gesetzt, jetzt die ganze Bandbreite der Prävention verstärkt in den Fokus und damit in das Licht der Öffentlichkeit zu rücken. Patientenorganisationen wie die Deutschen ILCO, eine bundesweite Selbstorganisation u. a. für Stomaträger, die es seit 40 Jahren gibt, leisten nachhaltig einen ganz entscheidenden Beitrag zur Unterstützung von Stomaträgern und Menschen mitDarmkrebs, wie im Beitrag von Herrn Grohmann, dem derzeitigen Vorsitzenden, und Frau Haß vorgestellt.
Die Themen des Schwerpunkthefts belegen ihre Größenordnung wie auch die Rolle in der Versorgung Betroffener.

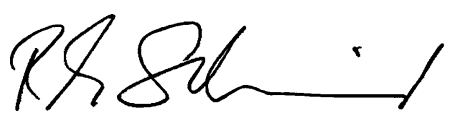

Prof. Dr. R. M. Schmid

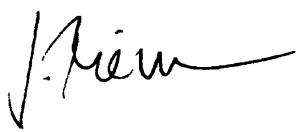

Prof. Dr. J. F. Riemann

\section{Korrespondenzadresse}

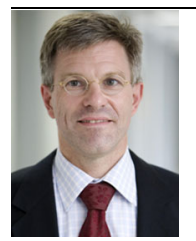

Prof. Dr. R. M. Schmid

II. Medizinische Klinik und Poliklinik Ismaninger Straße 22, 81675 München, Deutschland rolandm.schmid@mri.tum.de

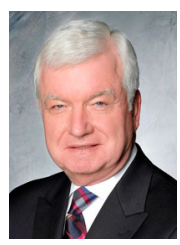

Prof. Dr. J. F. Riemann Medizinische Klinik C, Klinikum Ludwigshafen, Stiftung LebensBlicke Schuckertstr. 37, 67063 Ludwigshafen, Deutschland riemannj@garps.de

Interessenkonflikt. R.M. Schmid und J.F. Riemann geben an, dass kein Interessenkonflikt besteht. 